\title{
Efficacy of statins in patients with diabetic nephropathy: a meta-analysis of randomized controlled trials
}

\author{
Xue Shen ${ }^{1 \dagger}$, Zhongwen Zhang ${ }^{2 \dagger}$, Xiaoqian Zhang ${ }^{2}$, Junyu Zhao ${ }^{2}$, Xiaojun Zhou ${ }^{2}$, Qinglei Xu ${ }^{1}$, Hongxia Shang ${ }^{2}$, \\ Jianjun Dong ${ }^{3}$ and Lin Liao ${ }^{2^{*}}$
}

\begin{abstract}
Background: The effects of statins in patients with diabetic nephropathy are controversial. With increasing interest in the potential therapeutic role of statins in diabetic nephropathy, it is essential to evaluate its real effects.

Methods: PubMed, EMBASE, Web of Science databases, Cochrane Central Register of Controlled Trials and China National Knowledge Infrastructure were systematically searched for randomized controlled trials (RCTs) of statins in patients with diabetic nephropathy.

Results: Fourteen trials with 2866 participants were included in our meta-analysis. Compared with placebo, albuminuria and urinary albumin excretion rates in the statin group were reduced by 0.46 [95\% confidence interval $(\mathrm{Cl}),-0.68$ to $-0.25, P<0.0001]$ and $1.68(95 \% \mathrm{Cl},-3.23$ to $-0.12, P=0.03)$, respectively. The reduction of albuminuria was greater in patients of type 2 diabetes mellitus with diabetic nephropathy [standardized mean difference (SMD), $-0.56 ; 95 \% \mathrm{Cl}$, -0.80 to $-0.32, P<0.00001$ ] and the decrease was significant during the 1 to 3 years period of statin therapy (SMD, $-0.57 ; 95 \% \mathrm{Cl},-0.95$ to $-0.19, P=0.003)$. Subgroup analysis demonstrated the effects of statins were much stronger in subjects with pathologic albuminuria: change of $-0.71(95 \% \mathrm{Cl},-1.09$ to $-0.33, P=0.0003)$ for those with urinary protein excretion 30 to $300 \mathrm{mg} /$ day, -0.37 (95\% Cl, -0.67 to $-0.06, P=0.02$ ) for those with excretion more than $300 \mathrm{mg} /$ day and $-0.29(95 \% \mathrm{Cl},-0.78$ to $0.21, P=0.26)$ for those with excretion less than $30 \mathrm{mg} /$ day. In contrast, statins did not significantly reduce estimated glomerular filtration rate, serum creatinine and blood urea nitrogen levels.
\end{abstract}

Conclusions: Statins decrease the albuminuria and urinary albumin excretion rates significantly. The efficacy of statins on renal function is time dependent and better in type 2 diabetic patients with nephropathy.

Keywords: Statins, Diabetic Nephropathy, Meta-analysis

\section{Background}

According to the International Diabetes Federation [1], it is projected that the number of people with diabetes worldwide will increase from 382 million in 2013 to 592 million by 2035. Diabetic nephropathy (DN) is one of the most common and serious chronic complication of diabetes and it is the leading cause of end-stage renal disease [2]. However, beyond angiotensin II-receptor

\footnotetext{
* Correspondence: liaolin009@sina.com

${ }^{\dagger}$ Equal contributors

²Department of Medicine, Division of Endocrinology, Shandong Provincial

Qianfoshan Hospital, Shandong University, No.16766, Jingshi Road, Lixia

District, Jinan 250014, Shandong Province, China

Full list of author information is available at the end of the article
}

blockers (ARB) and angiotensin-converting enzyme inhibitors (ACEI), therapeutic options to block the progression of diabetic nephropathy are limited and other strategies to preserve kidney function are needed.

A number of potential mechanisms for kidney damage in DN have been identified. Hyperlipidemia may play an important role in the progression of $\mathrm{DN}$ and it may impair the messangial cells through its lipotoxicity or by promoting intrarenal atherosclerosis [3-5]. Statin, 3hydroxy-3 methylglutaryl coenzyme A (HMG CoA) reductase inhibitor, is a kind of antihyperlipidemic drug that used worldwide for its strong low-density lipoprotein cholesterol (LDL-C)-lowering effects and established 
safety. Recently, there are growing studies suggested that statins may offer renoprotective effects and beneficial effect on pathologic albuminuria and decrease the reduction of estimated glomerular filtration rate (eGFR) [6-8]. However, some trials $[9,10]$ failed to demonstrate that statin improve eGFR.

To assess whether statins have beneficial effects on renal outcomes in diabetic nephropathy, we performed this meta-analysis to investigate the potential therapy of statins in patients with diabetic nephropathy.

\section{Methods}

\section{Literature search}

We conducted a search of PubMed, EMBASE, Web of Science databases, Cochrane Central Register of Controlled Trials and China National Knowledge Infrastructure (CNKI). All relevant articles were published in English and Chinese. The following Medical Subject Headings (MeSH) and text words were used: Hydroxymethylglutaryl-CoA reductase inhibitors, atorvastatin, simvastatin, rosuvastatin, pravastatin, lovastatin, fluvastatin, cerivastatin, mevastatin, pitavastatin, statin, kidney, renal, diabetic nephropathy, randomized controlled trial (RCT), controlled clinical trial and random allocation. We also searched the additional trials at the trial register centres (http://www.clinicaltrials.gov). Clinical trials were included if the following criterias were met: (1) Primary study of statins versus control (placebo or usual care); (2) Diabetic nephropathy patients with type 1 and type 2 diabetes mellitus at least 18 years old without pregnancy; (3) Patients with diabetic nephropathy in experimental group were defined as those who used statins, regardless of dosages, mode of administration or treatment duration; (4) RCT design; (5) Report of baseline and the end of follow-up data on renal function [estimated glomerular filtration rate (eGFR), urinary albumin excretion rates (UAER), serum creatinine (Scr), blood urea nitrogen (BUN) or albuminuria). Exclusion criteria included: (1) Kidney damage due to diseases other than type 1 or type 2 diabetes. (2) The final stage of diabetic nephropathy or endstage-renal disease (ESRD), defined as onset of renal replacement therapy or death attributed to diabetic nephropathy.

\section{Study selection and data extraction}

Two reviewers independently screened abstracts according to the inclusion criteria, and disagreements between reviewers were resolved by consensus. We developed a data extraction sheet based on the Cochrane Consumers and Communication Review Group's data extraction template. One reviewer extracted the following data from included studies and the second reviewer verified the extracted data. Disagreements were resolved by discussion between the two reviewers. If an agreement could not be reached between two reviewers, a third author would decide. Information extracted included: (1) characteristics of trial subjects (including age, sex ratio, duration of diabetes and baseline value of renal function) and the trial's inclusion/exclusion criteria; (2) type of intervention (including dosage, duration and frequency); and (3) type of outcome and measurement.

\section{Statistical analysis}

The primary outcome was the change of albuminuria from the baseline. Other outcomes include: change from baseline in eGFR, UAER, Scr, BUN. The meta-analysis with fixed-model or random-model was performed by weighted mean difference (WMD), standardized mean difference (SMD) and $95 \%$ confidence interval (CI) for outcome of continuous variables. Subgroup analysis by characteristics of patients (i.e., ethnicity, stage of diabetes nephropathy) and study design (i.e., whether ACEI/ARB was used or not) were performed. $I^{2}$ was calculated as an index of heterogeneity between studies. If $I^{2}$ was higher than $50 \%$, the sensitive analysis should be performed to find out the source of heterogeneity and to assess whether the results could be significantly influenced.

\section{Quality assessment and publication bias}

Study quality and bias risk were assessed via predefined categories: randomization, allocation concealment, quality of blinding (participants,personnel and outcome assessment), withdrawal and loss and reporting bias. Two reviewers independently determined these items. Sensitive analysis was performed in studies with low quality. The analyses were performed using Review Manager 5.2 (Cochrane Collaboration, http://www.cochrane.org).

\section{Results}

\section{Search results and study characteristics}

Initially, 929 potentially relevant articles were identified. After adjusting for duplicates, 603 studies remained, consisting of 134 potentially relevant studies and 469 studies that were excluded after reviewing titles and abstracts. Of 134 potentially relevant studies, 120 failed to match the inclusion criteria. Finally 14 articles [9-22] with a total of 2866 participants were included in this meta-analysis (Fig. 1). Of these, 3 studies [20-22] were reported in Chinese, 11 were in English [9-19]. Among the final 14 studies, 7 were conducted in Caucasians and others in Asians. The most commonly diabetic nephropathy included in this meta-analysis was diabetic nephropathy with type 2 diabetes mellitus. Ten studies provided data of albuminuria, 5 of eGFR, 5 of UAER, 4 of Scr and 2 of BUN. Eight different statins involved in this study, including simvastatin [12, $13,15,17,22]$, atorvastatin $[9,11,20,21]$, pitavastatin [18], lovastatin [14], cerivastatin [19], rosuvastatin [10] and pravastatin [16]. The study period ranged from approximately 3 months to 2 years. ACEI or ARB were used in 


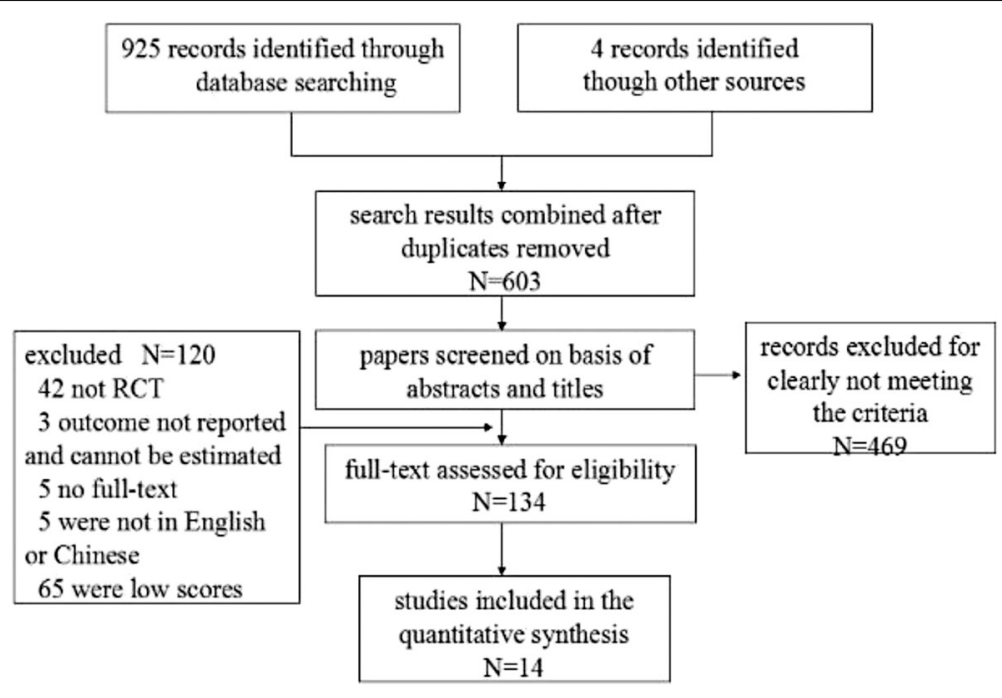

Fig. 1 Flow diagram of study selection

studies, except for 3 studies $[11,15,17]$. The characteristics of the included studies were shown in Tables 1 and 2. Among them, 7 studies $[9,10,12,14,20-22]$ mentioned the specific randomized method, the others referred "random" but did not mention the detail. Nine studies [9, 11-13, 15-19] were double-blinded. Detail was shown in Fig. 2.

\section{Quantitative data analysis}

\section{Effect of statins on albuminuria}

Pooled data from 10 studies [11-17, 19, 21, 22] (20 groups) showed a statistical decrease in albuminuria compared with that in control group (SMD, $-0.46 ; 95 \%$ CI, -0.68 to $-0.25 ; P<0.0001$ ), and the standardized mean difference in change from baseline was -0.71 (95\% CI, -1.09 to $-0.33 ; P=0.0003 ; I^{2}=33 \%$ ) for those with excretion of 30 to $299 \mathrm{mg} / \mathrm{d}$; and -0.37 (95\% CI, -0.67 to $-0.06 ; P=0.02 ; I^{2}=0 \%$ ) for those with excretion of 300 or greater (Fig. 3). Although the statins were not the same subtype, there was no significant heterogeneity among all trials in our study $\left(I^{2}=24 \%\right)$.

Statin did not decrease the urine albumin for those with excretion less than $30 \mathrm{mg} / \mathrm{d}$ (SMD, $-0.29 ; 95 \% \mathrm{CI},-0.78$ to $0.21 ; P=0.26$, Fig. 3). In statin treated group, there was

Table 1 Fifteen randomized, placebo-controlled trials assessing the effect of statins on renal outcomes in diabetic nephropathy

\begin{tabular}{|c|c|c|c|c|c|c|c|c|c|c|c|}
\hline \multirow[t]{2}{*}{ Studies } & \multirow[t]{2}{*}{ Country } & \multirow[t]{2}{*}{ Intervention } & \multicolumn{2}{|c|}{$\begin{array}{l}\text { Sample } \\
\text { sizes (n) }\end{array}$} & \multicolumn{2}{|c|}{$\begin{array}{l}\text { Use of ACEl or } \\
\text { ARB (\%) }\end{array}$} & \multicolumn{2}{|c|}{$\begin{array}{l}\text { Mean age } \\
\text { (years) }\end{array}$} & \multicolumn{2}{|c|}{$\begin{array}{l}\text { Duration of diabetic } \\
\text { nephropathy, (years) }\end{array}$} & \multirow[t]{2}{*}{$\begin{array}{l}\text { Follow-up } \\
\text { (months) }\end{array}$} \\
\hline & & & statin & control & statin & control & statin & control & statin & control & \\
\hline CARDS 2009 [9] & UK & Atorvastatin, $10 \mathrm{mg} / \mathrm{d}$ & 1154 & 1159 & 44.6 & 43.6 & 61.5 & 61.8 & - & - & 24 \\
\hline Masanori 2011 [10] & Japan & Rosuvastatin, $2.5-10 \mathrm{mg} / \mathrm{d}$ & 52 & 52 & 100 & 100 & 64.5 & 64.9 & - & - & 6 \\
\hline Dalla 2003 [11] & Italy & Atorvastatin $10 \mathrm{mg} / \mathrm{d}$ & 12 & 13 & 0 & 0 & 66 & 63 & 10 & 9 & 12 \\
\hline Linda 2001 [12] & USA & Simvastatin, $10 \mathrm{mg} / \mathrm{d}$ & 19 & 20 & 5 & 15 & 33.3 & 31.0 & 22.8 & 20.8 & 18 \\
\hline E. Hommel 1992 [13] & Denmark & Simvastatin, $10-20 \mathrm{mg} / \mathrm{d}$ & 12 & 9 & 67 & 89 & 41 & 35 & 27 & 27 & 3 \\
\hline Lam 1995 [14] & China & Lovastatin, 30 mg/d & 16 & 18 & 12.5 & 16.7 & 58.9 & 53.9 & - & - & 24 \\
\hline S.Nielsen 1993 [15] & Denmark & Simvastatin $10-20 \mathrm{mg} / \mathrm{d}$ & 8 & 10 & 0 & 0 & 65 & 65 & 10.2 & 10.9 & 9 \\
\hline Zhang 1995 [16] & Belgium & Pravastatin, 20 mg & 10 & 10 & - & - & 43 & 43 & - & - & 3 \\
\hline Giancarlo 1997 [17] & Italy & Simvastatin, 20 mg/d & 10 & 9 & 0 & 0 & 60 & 62 & - & - & 12 \\
\hline Tsukasa 2005 [18] & Japan & Pitavastatin, 1 mg/d & 10 & 10 & - & - & 51 & 49 & 13 & 12 & 12 \\
\hline Tsukasa 2001 [19] & Japan & Cerivastatin, $0.15 \mathrm{mg} / \mathrm{d}$ & 30 & 30 & - & - & 58 & 55 & - & - & 6 \\
\hline Wu 2013 [20] & China & Atorvastatin, 20 mg/d & 39 & 39 & 100 & 100 & 55.15 & 55.33 & 5.18 & 4.82 & 6 \\
\hline Du 2015 [21] & China & Atorvastatin, 20 mg/d & 26 & 26 & 100 & 100 & 56 & 57 & 10 & 10 & 3 \\
\hline Xiang 2005 [22] & China & Simvastatin, $20 \mathrm{mg} / \mathrm{d}$ & 32 & 31 & 100 & 100 & 50 & 49 & 15 & 14 & 6 \\
\hline
\end{tabular}

USA the United States of America, UK United Kingdom, T2DM type 2 diabetes mellitus, T1DM type 1 diabetes mellitus, -not report 
Table 2 Characteristics of the 14 randomized controlled trials Included in the meta-analysis

\begin{tabular}{|c|c|c|c|c|c|c|}
\hline Study or author & $\begin{array}{l}\text { Baseline } \\
\text { LDL-C Level, } \\
\text { (mg/dl) }\end{array}$ & $\begin{array}{l}\text { Change in } \\
\mathrm{LDL}-\mathrm{C}^{\mathrm{a}} \\
(\mathrm{mg} / \mathrm{dl})\end{array}$ & $\begin{array}{l}\text { Baseline } \\
\mathrm{HDL}-\mathrm{C} \text { Level, } \\
\text { (mg/dl) }\end{array}$ & $\begin{array}{l}\text { Change in } \\
\mathrm{HDL}-\mathrm{C}^{\mathrm{a}} \text {, } \\
(\mathrm{mg} / \mathrm{dl})\end{array}$ & $\begin{array}{l}\text { Baseline } \\
\text { Triglyceride } \\
\text { Level, (mg/dl) }\end{array}$ & $\begin{array}{l}\text { Change in } \\
\text { Triglyceride } \\
\text { (mg/dl) }\end{array}$ \\
\hline CARDS 2009 [9] & - & - & - & - & - & - \\
\hline Masanori 2011 [10] & 137 & -54 & 49 & +4 & 162 & -32 \\
\hline Dalla 2003 [11] & 149 & -41 & 55 & +1 & 162 & -32 \\
\hline Linda 2001 [12] & 125.5 & -28.3 & 50.9 & +2.2 & 76 & -9.5 \\
\hline E. Hommel 1992 [13] & 162.54 & -61.92 & 57.7 & +1.55 & 120.5 & +11.52 \\
\hline Lam 1995 [14] & 166.4 & -50.31 & 42.57 & -0.39 & 194.92 & -17.72 \\
\hline S.Nielsen 1993 [15] & 170.28 & -58.05 & 48.76 & -0.39 & 204.7 & -20.4 \\
\hline Zhang 1995 [16] & 123 & -23 & 62 & +1 & 105 & -12 \\
\hline Giancarlo 1997 [17] & 181.89 & -54.18 & 50.31 & 0 & 141.8 & -26.6 \\
\hline Tsukasa 2005 [18] & - & - & - & - & - & - \\
\hline Tsukasa 2001 [19] & 208 & -62 & 22 & +16 & 202 & -42 \\
\hline Wu 2013 [20] & 171.05 & -61.15 & - & - & 225.9 & -65.6 \\
\hline Du 2015 [21] & - & - & - & - & - & - \\
\hline Xiang 2005 [22] & 166.41 & -19.35 & 54.18 & +7.74 & 221.5 & -17.72 \\
\hline
\end{tabular}

In statin group;-:not report

statistically significant reduction in albuminuria in the T2DM with diabetic nephropathy (SMD,-0.56; $95 \% \mathrm{CI}$,0.80 to $-0.32 ; P<0.00001, I^{2}=14 \%$ ), while no significant improvement in diabetic nephropathy of T1DM subgroup (SMD, $-0.11 ; 95 \% \mathrm{CI},-0.52$ to $0.50 ; I^{2}=0 \% ; P=0.97$, Fig. 4). A greater decrease in albuminuria was observed in patients received statin therapy for 1 to 3 years (SMD, $-0.57 ; 95 \% \mathrm{CI},-0.95$ to $-0.19, P=0.002)$ compared with those $<1$ year (SMD;-0.41, $95 \% \mathrm{CI},-0.67$ to $-0.15, P=$ 0.003 ; Table 3). However, there was no significant difference between Asians (SMD $-0.46,95 \% \mathrm{CI}-0.80$ to -0.12 ) and Caucasians population (SMD $-0.54,95 \% \mathrm{CI}-0.82$ to -0.27 ; Table 3 ).

Therefore, the results suggested that statins can reduce albuminuria significantly in patients of T2DM with diabetic nephropathy. And the beneficial effect of statins on renal function are significantly better in those with statin therapy longer than one year than that of less than one year.

\section{Effect of statins on estimated glomerular filtration rate (eGFR)}

Six studies [9, 10, 13-15, 17] enrolled, including 2509 participants with eGFR $>60 \mathrm{ml} / \mathrm{min} / 1.73 \mathrm{~m}^{2}$. Statins did not improve eGFR significantly in most studies and the change in the SMD of eGFR was 0.49 (95\% CI, -0.06 to $\left.1.03, P=0.08, I^{2}=0 \%\right)$. Furthermore, different types of diabetic nephropathy, ethnicity, baseline of eGFR, treatment duration and dose of statins also did not influence eGFR in patients with statin and control therapy (Table 3).

\section{Effect of statins on urinary albumin excretion rates (UAER)}

The effect of statins on UAER was favorable in 5 studies $[15,16,18,20,22]$. Overall, the change in the SMD for UAER was -1.68 (95 \% CI, -3.23 to $-0.12, P=0.03$; Fig. 5) which indicated that compared with placebo, there was statistically significant reduction in UAER in

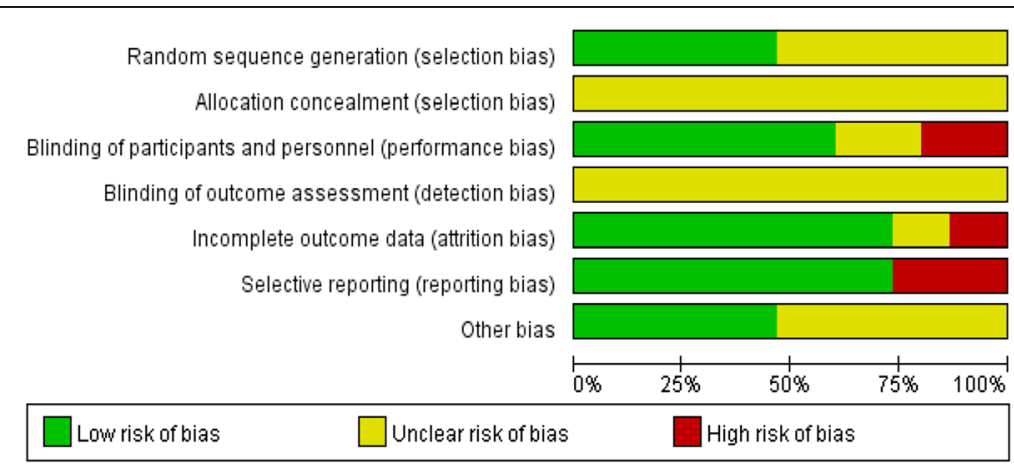

Fig. 2 Methodological quality of the included studies 


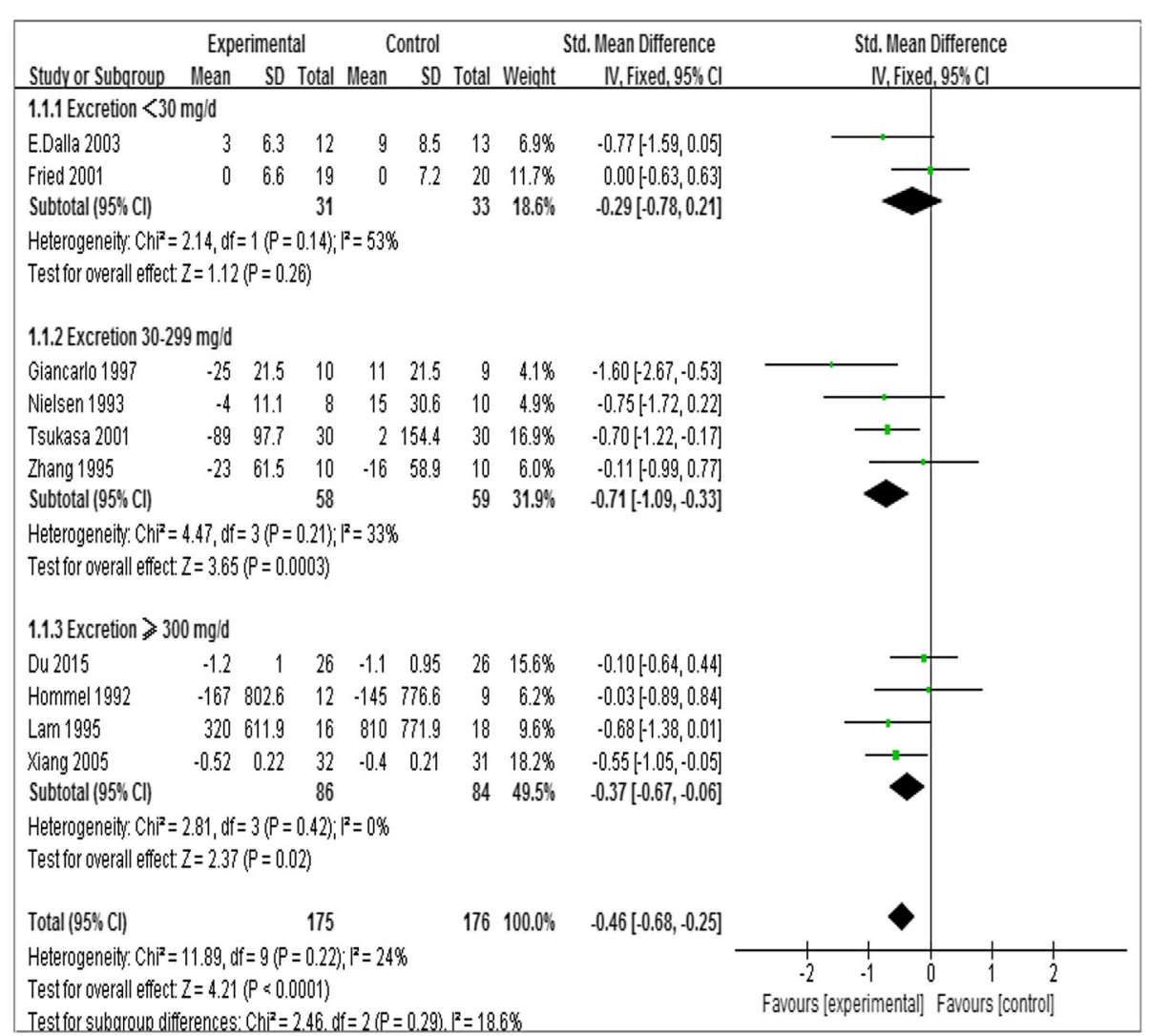

Fig. 3 Forest plots of albuminuria after statins or placebo therapy in patients with albuminuria $<30 \mathrm{mg} /$ day, 30 to $300 \mathrm{mg} /$ day, and $>300 \mathrm{mg} / \mathrm{day}$

statin-treated group. Because of the heterogeneity (heterozygosity test, $\mathrm{Chi}^{2}=56.01, \quad P<0.00001, I^{2}=$ $93 \%$ ), we conducted a subgroup analysis and sensitivity analysis. In subgroup analysis of ethnicity, stage of diabetic nephropathy, treatment duration and dose of statins, the heterogeneity still existed (Table 3). When the trials by Nielsen et al. (1993) [15], Zhang et al. (1995) [16] and Wu et al. (2013) [20] were removed, the heterogeneity disappeared (the $I^{2}$ reduced from $93 \%$ to $0 \%, P$ from $<0.00001$ to 0.76 ). The fixedeffect model was used to merge SMD values and the pooled SMD was -1.16 (95\% CI, -1.63 to $-0.69, P<$ $0.00001)$, which indicated that statins reduced UAER significantly in patients with DN.

\section{Effect of statins on serum creatinine (Scr)}

We identified 4 randomized controlled trials [19-22] (253 participants) for the effect of statins on Scr. Because of the heterogeneity (heterozygosity test, $\mathrm{Chi}^{2}=$ 64.91, $P<0.00001, I^{2}=95 \%$ ), we removed 1 study at a time to identify the source of heterogeneity. When the Study by Tsukasa et al. (2001) [19] was removed, the heterogeneity was no longer existence, which showed that the heterogeneity may came from the country difference of patients in the study. The fixed-effect model was used to merge SMD values and the pooled SMD was -0.10 (95\%CI, -0.38 to $0.19 ; P=0.50$; Table 3 ), which means statins may have no effect on the Scr in patients with DN.

\section{Effect of statins on blood urea nitrogen (BUN)}

The result showed that there was no statistically difference in BUN in statin group compared with that in control group (SMD, -0.26 ; $95 \% \mathrm{CI},-0.64$ to 0.13 ; $P=0.20)$ and the heterogeneity among trials was not significant $\left(I^{2}=0 \%\right.$, Table 3$)$.

\section{Assessment of publication bias}

Publication biases were examined by funnel plot and no significant publication bias was found among studies included in our meta-analysis (Fig. 6).

\section{Subgroup and sensitivity analysis}

Because of study heterogeneity, subgroup and metaregression analyses were conducted by ethnicity, baseline of albuminuria, treatment duration, and dose of statins (Table 3). However, the results of statin on renal function were not influenced. In consideration of the different baseline of UAER, sensitivity analysis was performed to assess the impact of every study on the overall 


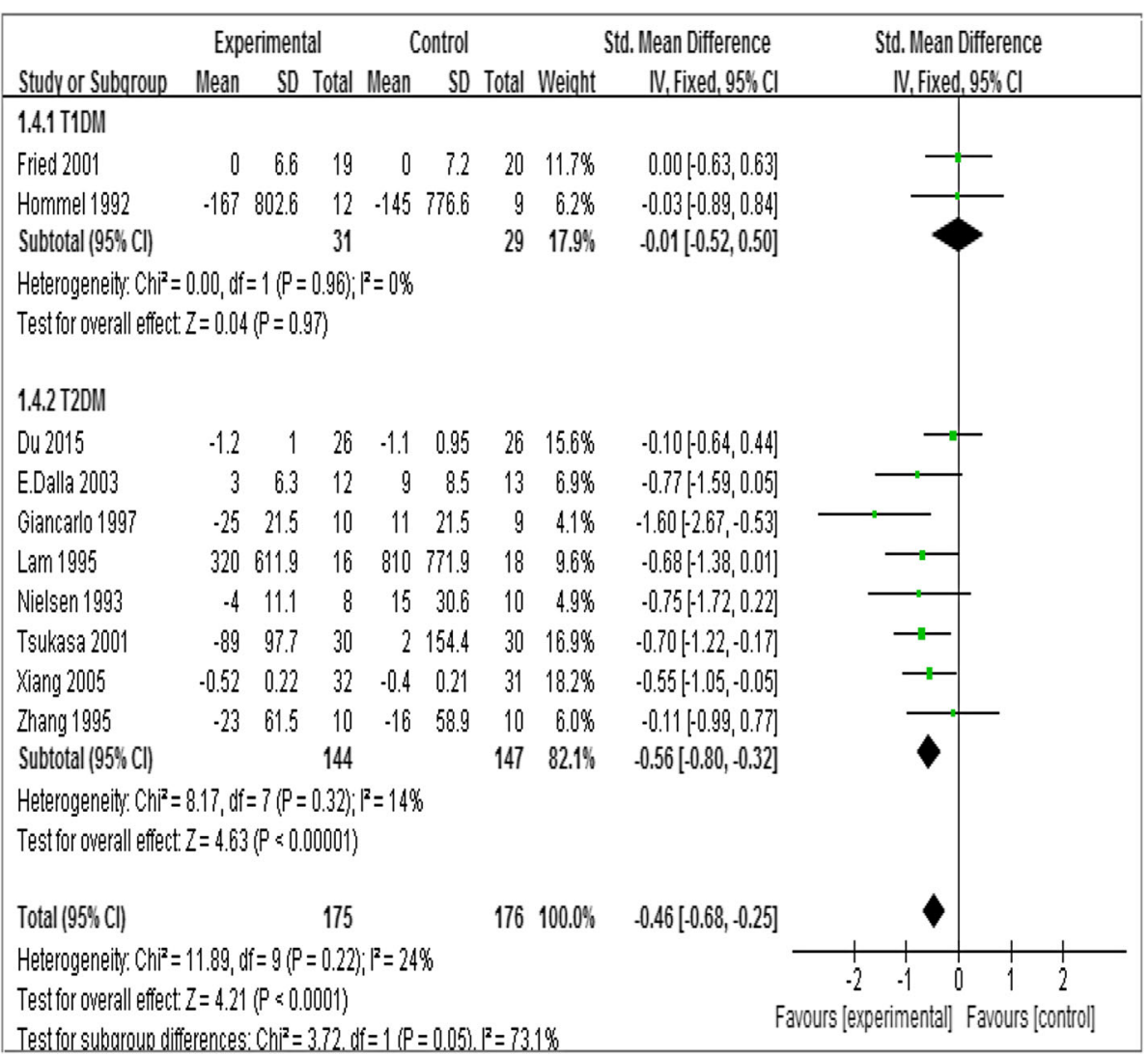

Fig. 4 Forest plots of albuminuria after statins or placebo therapy in nephropathy patients with T1DM or T2DM

conclusions. After the three trials by Nielsen et al. (1993) [15], Zhang et al. (1995) [16] and Wu et al. (2013) [20] were eliminated, the heterogeneity test (the $I^{2}$ reduces from $93 \%$ to $0 \%, P$ from $<0.00001$ to 0.76 ) indicated that baseline of UAER may be a source of heterogeneity (Fig. 7).

\section{Discussion}

Statins are frequently used to improve lipid profile and they are also reported to reduce cardiovascular events [23], albuminuria [6] and diabetic glomerulosclerosis in experimental animals [24]. However, the efficacy of statins in improving renal function in patients with diabetic nephropathy is still controversial. To answer this question, we did this study and found that statins could reduce the albuminuria and UAER compared with that in control groups. The beneficial effect of statins on renal function is time dependent and better in type 2 diabeties with nephropathy.

As we all know, the degree of albuminuria is a risk factor for renal failure [25]. Some studies have demonstrated a benefit of statins on albuminuria $[6,26,27]$, while others failed to indicate such an effect $[16,28]$. Our meta-analysis suggested an overall significant decrease of albuminuria after statin therapy (decrease by $0.46, P<0.0001$ ), with the greater improvement of albuminuria among studies with greater baseline albuminuria. Notably, our results are consistent with the meta-analysis performed by Kevin Douglas et al. [29]. The beneficial effect of statins on albuminuria may be potentially explained by cholesterol dependent effects and cholesterol independent effects [30]. Keane et al. [31] have showed that dyslipidemia contributes to glomerular and interstitial injury and the severity of the hypercholesterolemia correlates with albuminuria. In addition, statins may have other cholesterol-independent renoprotective actions, such as reducing neutrophil and macrophage infiltration, up-regulating endothelial nitric oxide (NO) synthase, inhibition of renal cell proliferation, antifibrotic and antioxidant effects, and down-regulation of inflammatory cytokines [32]. Researches suggested that statins reduce albuminuria at least in part by reducing inflammation and fibrosis in the renal interstitium, seemingly through actions on monocyte chemotactic protein-1 and transforming growth factor- $\beta$ (TGF- $\beta$ ) $[33,34]$.

A recent review [32] reported that the benefit of statins may depend on the duration of treatment. We also investigated the effect of statin treatment less than 1 year and between 1 and 3 years. A greater decrease of albuminuria was observed in patients received statin therapy for 1 to 3 years compared with those for less 
Table 3 Meta-analysis of the effect of statins on renal outcomes in diabetes

\begin{tabular}{|c|c|c|c|c|c|c|}
\hline \multirow[b]{2}{*}{ Category } & \multirow[b]{2}{*}{$\mathrm{N}$} & \multirow{2}{*}{$\begin{array}{l}\text { Subjects } \\
\text { cases/controls }\end{array}$} & \multicolumn{2}{|c|}{ Heterogeneity } & \multirow[b]{2}{*}{$\operatorname{SMD}(95 \% \mathrm{Cl})$} & \multirow[b]{2}{*}{$Z$ test } \\
\hline & & & $\overline{\mathrm{Ph}}$ & 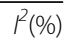 & & \\
\hline \multicolumn{7}{|l|}{ Albuminuria } \\
\hline 1. Overall & 10 & $175 / 176$ & 0.22 & 24 & $-0.46(-0.68,-0.25)$ & $z=4.21, p_{z}<0.0001$ \\
\hline \multicolumn{7}{|l|}{ 2. Adjustment by ethnicity } \\
\hline Caucasian & 6 & $71 / 71$ & 0.12 & 43 & $-0.41(-0.75,-0.07)$ & $z=2.36, p_{z}=0.02$ \\
\hline Asian & 4 & $104 / 105$ & 0.41 & 0 & $-0.50(-0.77,-0.22)$ & $z=3.51, p_{z}=0.0004$ \\
\hline \multicolumn{7}{|c|}{ 3. Adjustment by subtypes of diabetes with diabetic nephropathy } \\
\hline T1DM & 2 & $31 / 29$ & 0.96 & 0 & $-0.01(-0.52,0.50)$ & $z=0.04, p_{z}=0.97$ \\
\hline T2DM & 8 & $144 / 147$ & 0.31 & 14 & $-0.56(-0.80,-0.32)$ & $z=4.63, p_{z}<0.00001$ \\
\hline \multicolumn{7}{|c|}{ 4. Adjustment by baseline of albuminuria } \\
\hline$<30 \mathrm{mg} / \mathrm{d}$ & 2 & $31 / 33$ & 0.14 & 53 & $-0.29(-0.78,0.21)$ & $z=1.12, p_{z}=0.26$ \\
\hline $30-299 \mathrm{mg} / \mathrm{d}$ & 4 & $58 / 59$ & 0.21 & 33 & $-0.71(-1.09,-0.33)$ & $z=3.65, p_{z}=0.0003$ \\
\hline$\geq 300 \mathrm{mg} / \mathrm{d}$ & 4 & $86 / 84$ & 0.42 & 0 & $-0.37(-0.67,-0.06)$ & $z=2.37, p_{z}=0.02$ \\
\hline \multicolumn{7}{|c|}{ 5. Adjustment by treatment duration } \\
\hline$<1$ year & 6 & $118 / 116$ & 0.50 & 0 & $-0.41(-0.67,-0.15)$ & $z=3.09, p_{z}=0.002$ \\
\hline $1 \sim 3$ years & 4 & $57 / 60$ & 0.07 & 58 & $-0.57(-0.95,-0.19)$ & $z=2.94, p_{z}=0.003$ \\
\hline \multicolumn{7}{|c|}{ 6. Adjustment by dose of statins } \\
\hline low-intensity statins & 3 & $61 / 63$ & 0.18 & 41 & $-0.48(-0.84,-0.12)$ & $z=2.61, p_{z}=0.009$ \\
\hline moderate-intensity statins & 2 & 20/19 & 0.27 & 16 & $-0.35(-0.99,0.30)$ & $z=1.05, p_{z}=0.29$ \\
\hline high-intensity statins & 5 & $94 / 94$ & 0.13 & 44 & $-0.47(-0.77,-0.18)$ & $z=3.15, p_{z}=0.002$ \\
\hline \multicolumn{7}{|l|}{ eGFR } \\
\hline 1. Overall & 6 & $1252 / 1257$ & 0.73 & 0 & $0.49(-0.06,1.03)$ & $z=1.75, p_{z}=0.08$ \\
\hline \multicolumn{7}{|l|}{ 2. Adjustment by ethnicity } \\
\hline Caucasian & 5 & $1184 / 1187$ & 0.48 & 0 & $0.48(-0.08,1.04)$ & $z=1.69, p_{z}=0.09$ \\
\hline Asian & 1 & $68 / 70$ & 0.75 & 0 & $0.64(-1.87,3.15)$ & $z=0.50, p_{z}=0.62$ \\
\hline \multicolumn{7}{|c|}{ 3. Adjustment by subtypes of diabetes with diabetic nephropathy } \\
\hline T1DM & 1 & $12 / 9$ & not $\bar{c}$ & & $-3.00(-17.22,11.22)$ & $z=0.41, p_{z}=0.68$ \\
\hline T2DM & 5 & $1240 / 1248$ & 0.64 & 0 & $0.49(-0.05,1.04)$ & $z=1.77, p_{z}=0.08$ \\
\hline \multicolumn{7}{|c|}{ 4. Adjustment by baseline of albuminuria } \\
\hline$<30 \mathrm{mg} / \mathrm{d}$ & 1 & $913 / 918$ & not $\mathrm{c}$ & & $0.34(-0.28,0.96)$ & $z=1.08, p_{z}=0.28$ \\
\hline $30-299 \mathrm{mg} / \mathrm{d}$ & 4 & $311 / 312$ & 0.52 & 0 & $1.03(-0.16,2.21)$ & $z=1.70, p_{z}=0.09$ \\
\hline$\geq 300 \mathrm{mg} / \mathrm{d}$ & 1 & $28 / 27$ & 0.56 & 0 & $0.73(-6.15,7.61)$ & $z=0.21, p_{z}=0.83$ \\
\hline \multicolumn{7}{|c|}{ 5. Whether combined with ACER or ARB } \\
\hline with $A C E I$ or $A R B$ & 4 & $1218 / 1220$ & 0.74 & 0 & $0.04(-0.04,0.12)$ & $z=0.89, p_{z}=0.37$ \\
\hline without ACEI or ARB & 2 & $18 / 20$ & 0.94 & 0 & $1.14(0.44,1.84)$ & $z=3.20, p_{z}=0.001$ \\
\hline \multicolumn{7}{|c|}{ 6. Adjustment by treatment duration } \\
\hline$<1$ year & 3 & $72 / 71$ & 0.47 & 0 & $0.69(-1.85,3.24)$ & $z=0.53, p_{z}=0.59$ \\
\hline $1 \sim 3$ years & 3 & $1180 / 1186$ & 0.56 & 0 & $0.48(-0.08,1.04)$ & $z=1.68, p_{z}=0.09$ \\
\hline \multicolumn{7}{|c|}{ 7. Adjustment by dose of statins } \\
\hline low-intensity statins & 3 & $1206 / 1211$ & 0.81 & 0 & $0.42(-0.13,0.98)$ & $z=1.51, p_{z}=0.13$ \\
\hline moderate-intensity statins & 2 & 20/19 & 0.27 & 19 & $3.07(-6.28,12.43)$ & $z=0.64, p_{z}=0.52$ \\
\hline high-intensity statins & 1 & $26 / 27$ & 0.80 & 0 & $2.76(-0.83,6.36)$ & $z=1.51, p_{z}=0.13$ \\
\hline
\end{tabular}


Table 3 Meta-analysis of the effect of statins on renal outcomes in diabetes (Continued)

\begin{tabular}{|c|c|c|c|c|c|c|}
\hline \multicolumn{7}{|c|}{ 8. Adjustment by baseline of eGFR } \\
\hline $60 \sim 89 \mathrm{ml} / \mathrm{min} / 1.73 \mathrm{~m}^{2}$ & 4 & $1234 / 1238$ & 0.94 & 0 & $0.43(-0.12,0.98)$ & $z=1.52, p_{z}=0.13$ \\
\hline$>=90 \mathrm{ml} / \mathrm{min} / 1.73 \mathrm{~m}^{2}$ & 2 & $18 / 19$ & 0.48 & 0 & $3.45(-0.40,7.30)$ & $z=1.76, p_{z}=0.08$ \\
\hline \multicolumn{7}{|l|}{ UAER } \\
\hline 1. Overall & 5 & $99 / 100$ & $<0.00001$ & 93 & $-1.68(-3.23,-0.12)$ & $z=2.12, p_{z}=0.03$ \\
\hline \multicolumn{7}{|l|}{ 2. Adjustment by ethnicity } \\
\hline Caucasian & 2 & $18 / 20$ & $<0.00001$ & 96 & $-25.99(-78.54,26.57)$ & $z=0.97, p_{z}=0.33$ \\
\hline Asian & 3 & $81 / 80$ & 0.0001 & 89 & $-1.78(-2.98,-0.57)$ & $z=2.88, p_{z}=0.004$ \\
\hline \multicolumn{7}{|c|}{ 3. Adjustment by baseline of albuminuria } \\
\hline $30-299 \mathrm{mg} / \mathrm{d}$ & 4 & $67 / 69$ & $<0.00001$ & 94 & $-2.17(-4.56,0.23)$ & $z=1.77, p_{z}=0.08$ \\
\hline$\geq 300 \mathrm{mg} / \mathrm{d}$ & 1 & $32 / 31$ & not applicable & & $-1.12(-1.65,-0.58)$ & $z=4.11, p_{z}<0.0001$ \\
\hline \multicolumn{7}{|c|}{ 4. Adjustment by treatment duration } \\
\hline$<1$ year & 4 & $89 / 90$ & $<0.00001$ & 95 & $-1.90(-3.91,0.11)$ & $z=1.85, p_{z}=0.06$ \\
\hline $1 \sim 3$ years & 1 & $10 / 10$ & not applicable & & $-1.29(-2.28,-0.31)$ & $z=2.58, p_{z}=0.010$ \\
\hline \multicolumn{7}{|l|}{ 5. Adjustment by dose of statins } \\
\hline low-intensity statins & 1 & $10 / 10$ & not applicable & & $-1.29(-2.28,-0.31)$ & $z=2.58, p_{z}=0.010$ \\
\hline moderate-intensity statins & 1 & $8 / 10$ & not applicable & & $-53.77(-73.67,-33.88)$ & $z=5.30, p_{z}<0.00001$ \\
\hline high-intensity statins & 3 & $81 / 80$ & $<0.00001$ & 93 & $-1.39(-2.87,0.09)$ & $z=1.84, p_{z}=0.07$ \\
\hline \multicolumn{7}{|l|}{ Scr } \\
\hline 1. Overall & 4 & $127 / 126$ & $<0.00001$ & 95 & $0.75(-0.52,2.03)$ & $z=1.15, p_{z}=0.25$ \\
\hline \multicolumn{7}{|c|}{ 2. Adjustment by baseline of albuminuria } \\
\hline $30-299 \mathrm{mg} / \mathrm{d}$ & 2 & $69 / 69$ & $<0.00001$ & 98 & $1.62(-1.91,5.15)$ & $z=0.90, p_{z}=0.37$ \\
\hline$\geq 300 \mathrm{mg} / \mathrm{d}$ & 2 & $58 / 57$ & 0.93 & 0 & $-0.05(-0.42,0.31)$ & $z=0.28, p_{z}=0.78$ \\
\hline \multicolumn{7}{|c|}{ 4. Adjustment by dose of statins } \\
\hline low-intensity statins & 1 & $30 / 30$ & not applicable & & $3.44(2.62,4.25)$ & $z=0.00, p_{z}=1.00$ \\
\hline high-intensity statins & 3 & $97 / 96$ & 0.93 & 0 & $-0.10(-0.38,0.19)$ & $z=0.98, p_{z}=0.33$ \\
\hline \multicolumn{7}{|l|}{ BUN } \\
\hline 1.Overall & 2 & $51 / 52$ & 0.88 & 0 & $-0.26(-0.64,0.13)$ & $z=1.29, p_{z}=0.20$ \\
\hline
\end{tabular}

Abbreviations: $N$ number of involved studies, $P h P$ values for heterogeneity of $Q$ test, $p_{z}<0.05$ indicate significant association, eGFR estimated Glomerular Filtration Rate, T1DM type 1 diabetes mellitus, T2DM type 2 diabetes mellitus, ACEI angiotensin-converting enzyme inhibitors, ARB Angiotensin II -receptor blockers, UAER urinary albumin excretion rates, $S c r$ serum creatinine, $B U N$ blood urea nitrogen

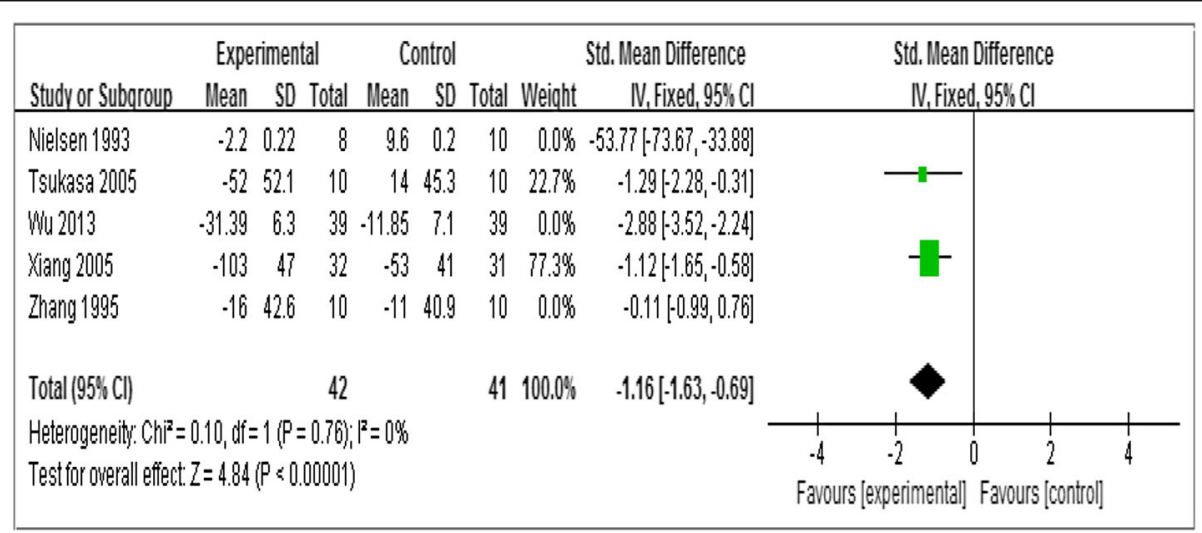

Fig. 5 Forest plots of UAER (ug/min) for statins versus placebo in patients with diabetic nephropathy 


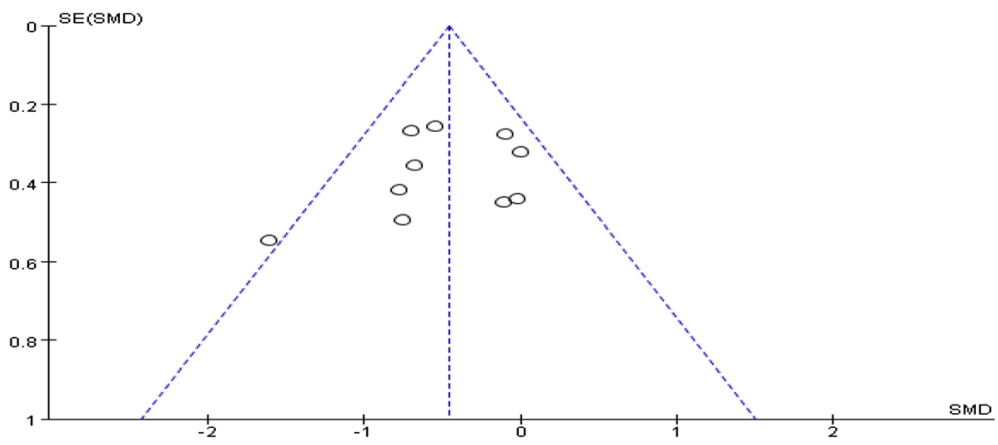

Fig. 6 Funnel plot of publication bias for the effects of statins on renal outcomes in diabetic nephropathy

than 1 year. It indicated that the beneficial effect of statins on albuminuria may depend on the duration of statin treatment. Additionally, our study found that statins can reduce albuminuria significantly in patients of T2DM with diabetic nephropathy. Diseaes progression, duration of statin therapy and improved renal blood flow are possible relevant factors [35, 36].

Some authors found statins may slow the decline in eGFR [37, 38]. Nikolic et al. [32] suggested an overall significant increase of GFR after statin therapy (increase by $0.29 \mathrm{ml} / \mathrm{min} / 1.73 \mathrm{~m}^{2}, p=0.04$ ), with the greatest GFR improvement after between 1 and 3 years of statin therapy $\left(0.50 \mathrm{ml} / \mathrm{min} / 1.73 \mathrm{~m}^{2} ; p<0.0001\right)$. However, our meta-analysis found that statins did not improve eGFR significantly. Just as Satirapoj said [30], as a post hoc analysis, using estimates of renal function, some limitations were observed in interpreting these data, so a small proportion of patients, who had DN, were included in this analysis, whereas our findings in the statin group revealed eGFR did not improve, but no significant decline was observed among DN subjects. Therefore, the available data on statin with eGFR in DN patients are still conflicting, because of possible outcome reporting bias.
Findings from our meta-analysis revealed that statins could reduce both albuminuria and the rate of progression of diabetic nephropathy. The benefits appear to supplement those derived from treatment with reninangiotensin system (RAS) inhibitors [10]. The pathogenesis of diabetic nephropathy is multifactorial and its precise mechanisms of action remain unclear. However, it is now widely accepted that the rate of functional deterioration correlates with the degree of the renal tubulointerstitial fibrosis [36]. Statins is effective in protecting against tubulointerstitial injury [10] and may slow down the progression of diabetic nephropathy, but this needs to be further validated in large-scale and long follow-up period randomized controlled trials.

As with any study, our meta-analysis had some limitations. Frist, the number of randomized controlled trials was small and only published data included. Second, the detection technique of albuminuria was different. Third, the results are heavily based on the findings of the CARDS trial, which represents more than $90 \%$ of the population of the meta-analysis. So, more clinical researches with larger sample, higher quality and strictly RCT study should be taken in the future.

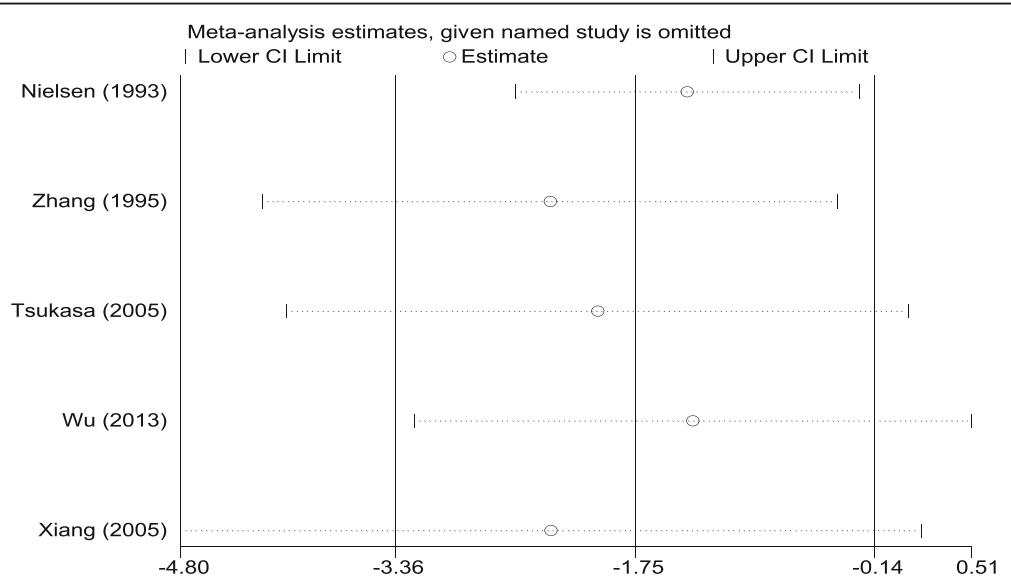

Fig. 7 Sensitivity analysis for the efficacy of statins on UAER in patients with diabetic nephropathy 


\section{Conclusion}

Statins reduce albuminuria and UAER significantly . The beneficial effect of statins on renal function is time dependent and better in type 2 diabeties patients with nephropathy. Our findings, though exciting, still require larger and high-quality studies to confirm the kidney benefit of statins in patients with diabetes.

\section{Acknowledgments}

This work was funded by National Natural Science Foundation of China Grants (81070637, 81570742), Natural Science Foundation of Shandong Province (No. Y2006C76, Y2008C73, ZR2010HM044).

\section{Funding}

The funding body of these National Natural Science Foundation of China Grants $(81070637,81570742)$ in the design of the study and collection, and in writing the manuscript played an important role. The funding of Natural Science Foundation of Shandong Province (No. Y2006C76, Y2008C73, ZR2010HM044) were crucial in analysis and interpretation of data.

\section{Availability of data and materials}

Data sharing not applicable to this article as no datasets were generated or analysed during the current study.

\section{Authors' contributions}

In this work, LL conceived and designed the systematic review protocol; SX $Z Z W, Z X Q, Z J Y, Z X J, X Q L, S H X$ and DJJ performed the study selection and data extraction; SX, ZZW, ZXQ, ZJY and ZXJ performed the statistical analyses; SX, ZZW and LL prepared the manuscript outlines and drafts. All authors contributed to the critical revision of manuscript drafts, as well as giving final approval for submitted manuscript content.

\section{Competing interests}

The authors declare that they have no competing interests.

\section{Consent for publication}

All the authors have agreed to publish this article.

\section{Ethics approval and consent to participate}

Not applicable.

\section{Author details \\ 'Department of Medicine, Shandong Provincial Qianfoshan Hospital, Shandong University of Traditional Chinese Medicine, Jinan 250014, China. 2Department of Medicine, Division of Endocrinology, Shandong Provincial Qianfoshan Hospital, Shandong University, No.16766, Jingshi Road, Lixia District, Jinan 250014, Shandong Province, China. ${ }^{3}$ Department of Medicine, Division of Endocrinology, Qilu Hospital of Shandong University, Jinan 250012, China.}

Received: 8 September 2016 Accepted: 7 October 2016 Published online: 12 October 2016

\section{References}

1. Shi Y, Hu FB. The global implications of diabetes and cancer. Lancet. 2014:383(9933):1947-8

2. Guo Z, Liu X, et al. Differential urinary glycoproteome analysis of type 2 diabetic nephropathy using 2D-LC-MS/MS and iTRAQ quantification. J Transl Med. 2015;13(1):371.

3. Toneli M, Moye L, Sacks FM, Cole T, Curhan GC. Effect of pravastatin on loss of renal fuction in people with moderate chronic renal insufficiency and cardiovascular disease. J Am Soc Nephrol. 2003;14:1605-13.

4. Guijaro C, Keane WF. Effects of lipids on the pathogenesis of progressive renal failure: role of 3-hydroxy-3-methylglutary coenzyme A reductase inhibitors in the prevention of glomerulosclerosis. Miner Electrolyte Metab. 1996;22(1-3):147-52.

5. Fried LF, Orchard TJ, Kasiske BL. Effect of lipid reduction on the progression of renal disease: A meta-analysis. Kidney Int. 2001;59:260-9.
6. Zhou S, Zhao P, Li Y, Deng T, Tian L, Li H. Renoprotective effect of atorvastatin on STZ-diabetic rats through attenuating kidney-associated dysmetabolism. Eur J Pharmacol. 2014;740:9-14.

7. Sandhu S, Wiebe $N$, et al. Statins for improving renal outcomes: a meta-analysis. J Am Soc Nephrol. 2006;17(7):2006-16.

8. Geng Q, Ren J, et al. Meta-analysis of the effect of statins on renal function. Am J Cardiol. 2014;114(4):562-70.

9. Colhoun HM, Betteridge DJ, Durrington PN, Hitman GA, Neil HA, Livingstone SJ, Charlton-Menys V, DeMicco DA, Fuller JH, CARDS Investigators. . Effects of atorvastatin on kidney outcomes and cardiovascular disease in patients with diabetes: an analysis from the Collaborative Atorvastatin Diabetes Study (CARDS). Am J Kidney Dis. 2009:54(5):810-9.

10. Abe M, Maruyama N, Okada K, Metsumoto S, Matsumoto K, Soma M. Effects of lipid-lowering therapy with rosuvastatin on kidney function and oxidative stress in patients with diabetic nephropathy. J Atheroscler Thromb. 2011; 18(11):1018-28.

11. Dalla Nora E, Passaro A, Zamboni PF, et al. Atorvastatin improves metabolic control and endothelial function in type diabetic patients: a placebocontrolled study. J Endocrinol Invest. 2003;26:73-8.

12. Fried LF, Forrest $K Y$, Ellis D, Chang $Y$, Silvers $N$, Orchard TJ. Lipid modulation in insulin-dependent diabetes mellitus: effect on microvascular outcomes. J Diabetes Complications. 2001;15(3):113-9.

13. Hommel E, Andersen P, Gall M-A, Nielsen F, Jensen B, Rossing P, Dyerberg J, Parving $\mathrm{H}-\mathrm{H}$. Plasma lipoproteins and renal function during simvastatin treatment in diabetic nephropathy. Diabetologia. 1992;35:447-51.

14. Lam KSL, Cheng IKP, Janus ED, et al. Cholesterol-lowering therapy may retard the progression of diabetic nephropathy. Diabetologia. 1995;38:604-9.

15. Nielsen S, Schmitz O, Molle N, et al. Renal function and insulin sensitivity during simvastatin treatment in Type 2 (non-insulin-dependent) diabetic patients with microalbuminuria. Diabetologia. 1993;36:1079-86.

16. Zhang A, Vertommen J, Van Gaal L, et al. Effects of pravastatin on lipid levels, in vitro oxidizability of non-HDL lipoproteins and microalbuminuria in IDDM patients. Diabetes Res Clin Pract. 1995;29:189-94.

17. Tonolo G, Calvia P, Ciccarese M, et al. Reduction of albumin excretion rate in normotensive microalbuminuric type 2 diabetic patients during longterm simvastatin treatment. Diabetes Care. 1997;20(12):1891-5.

18. Nakamura $T$, Ueda $Y$, Sugaya $T$, et al. Effect of pitavastatin on urinary livertype fatty acid-binding protein levels in patients with early diabetic nephropathy. Diabetes Care. 2005;28(11):2728-32.

19. Nakamura T, Ushiyama C, Hirokawa K, Osada S, Shimada N, Koide H. Effect of cerivastatin on urinary albumin excretion and plasma endothelin-1 concentrations in type 2 diabetes patients with microalbuminuria and dyslipidemia. Am J Nephrol. 2001;21(6):449-54.

20. Wu G, Gao X, Changlin M. Clinical Observe Effect of Atorvastatin Combined with Benazepril Treatment of Early Diabetic Nephropathy. China Academic J Electronic Publishing House. 2013;14(2):117-9.

21. Xiaoli D, Shen J, Ting L. Lipitor and emberd combined treat the blood lipids of diabetic nephropathy. Sichun MEdical Journal. 2015;36(3):404-6.

22. Qun X, Qiang Y, Ye D, et al. The split or combination of Simvastatin and bei treat diabetic nephropathy. China New Drugs. 2005;24(6):440-3.

23. Jabir NR, Siddiqui AN, Firoz CK, Md Ashraf G, Zaidi SK, Khan MS, Shakil S, Alama MN, Kamal MA, Tabrez S. Current Updates on Therapeutic Advances in the Management of Cardiovascular Diseases. Curr Pharm Des. 2016;22(5):566-71.

24. Vázquez-Pérez S, Aragoncillo P, de Las HN, et al. Atorvastatin prevents glomerulosclerosis and renal endothelial dysfunction in hypercholesterolaemic rabbits. Nephrol Dial Transplant. 2001:16 suppl 1:40-4.

25. Hunt LP, Short CD, Mallick NP. Prognostic indicators in patients presenting with the nephrotic syndrome. Kidney Int. 1988:34(3):382-8.

26. Geng Q, Ren J, Song J, Li S, Chen H. Meta-analysis of the effect of statins on renal function. Am J Cardiol. 2014;114(4):562-70.

27. Su X, Zhang L, Lv J, Wang J, Hou W, Xie X, Zhang H. Effect of Statins on Kidney Disease Outcomes: A Systematic Review and Meta-analysis. Am J Kidney Dis. 2016;67(6):881-92.

28. Sanguankeo A, Upala S, Cheungpasitporn W, Ungprasert P, Knight EL. Effects of Statins on Renal Outcome in Chronic Kidney Disease Patients: A Systematic Review and Meta-Analysis. PLoS One. 2015;10(7):e0132970.

29. Douglas K, O'Malley PG, Jackson JL. Meta-Analysis: The Effect of Statins on Albuminuria. Ann Intern Med. 2006;145(2):177-24. 
30. Satirapoj B, Promrattanakun A, Supasyndh O, Choovichian P. The Effects of Simvastatin on Proteinuria and Renal Function in Patients with Chronic Kidney Disease. Int J Nephrol. 2015;2015:485839.

31. Keane WF. The role of lipids in renal disease: future challenges. Kidney Int. 2000;75:527-31.

32. Nikolic D, Banach M, Nikfar S, Salari P, Mikhailidis DP, Toth PP, Abdollahi M, Ray KK, Pencina MJ, Malyszko J, Rysz J, Rizzo M, Lipid and Blood Pressure Meta-Analysis Collaboration Group. A meta-analysis of the role of statins on renal outcomes in patients with chronic kidney disease. Is the duration of therapy important? Int J Cardiol. 2013;168(6):5437-47.

33. Athyros VG, Kakafika Al, Karagiannis A, Mikhailidis DP. Do we need to consider inflammatory markers when we treat atherosclerotic disease? Atherosclerosis. 2008;200(1):1-12.

34. Zoja C, Corna D, Camozzi D, Cattaneo D, Rottoli D, Batani C, Zanchi C, Abbate M, Remuzzi G. How to fully protect the kidney in a severe model of progressive nephropathy: A multidrug approach. J Am Soc Nephrol. 2002;13:2898-908.

35. Athyros VG, Mikhailidis DP, Liberopoulos EN, et al. Effect of statin treatment on renal function and serum uric acid levels and their relation to vascular events in patients with coronary heart disease and metabolic syndrome. A subgroup analysis of the GREek Atorvastatin and Coronary heart disease Evaluation (GREACE) Study. Nephrol Dial Transplant. 2007;22(1):118-27.

36. Phillips AO, Steadman R. Diabetic nephropathy: the central role of renal proximal tubular cells in tubulointerstitial injury. Histol Histopathol. 2002;17(1):247-52.

37. Hou W, Lv J, Perkovic V, et al. Effect of statin therapy on cardiovascular and renal outcomes in patients with chronic kidney disease: a systematic review and metaanalysis. Eur Heart J. 2013;34(24):1807-17.

38. Verma A, Ranganna KM, Reddy RS, Verma M, Gordon NF. Effect of rosuvastatin on C-reactive protein and renal function in patients with chronic kidney disease. Am J Cardiol. 2005;96(9):1290-2.

\section{Submit your next manuscript to BioMed Central and we will help you at every step:}

- We accept pre-submission inquiries

- Our selector tool helps you to find the most relevant journal

- We provide round the clock customer support

- Convenient online submission

- Thorough peer review

- Inclusion in PubMed and all major indexing services

- Maximum visibility for your research

Submit your manuscript at www.biomedcentral.com/submit 